\title{
STEADY FLOW OF A SLIGHTLY VISCOELASTIC FLUID BETWEEN ROTATING SPHERES*
}

\author{
BY \\ WILLIAM E. LANGLOIS \\ IBM Research Laboratory, San Jose, California
}

1. Introduction. The steady flow of an incompressible, slightly non-Newtonian fluid of the type first studied by Rivlin and Ericksen [4] was considered in a previous paper [1]. It was assumed that the stress components $t_{i i}(i, j=1,2,3)$ in a Cartesian coordinate system $x_{i}$, at a point $x_{i}$ of the fluid, are related to the kinematic state of flow by the Cartesian tensor relation

$$
t_{i j}=-p \delta_{i i}+\mu A_{i j}^{(1)}+\epsilon P_{i j}(i, j=1,2,3),
$$

where $\epsilon$ is a small, dimensionless constant, $p$ is an undetermined pressure, $\mu$ is constant, and $P_{i j}$ is a symmetric tensor polynomial in $R$ kinematic tensors $A_{i j}^{(\gamma)}(\gamma=1,2, \cdots, R)$ defined by**

$$
\begin{gathered}
A_{i j}^{(1)}=\frac{\partial v_{i}}{\partial x_{i}}+\frac{\partial v_{i}}{\partial x_{i}}(i, j=1,2,3), \\
A_{i j}^{(\gamma+1)}=v_{p} \frac{\partial A_{i j}^{(\gamma)}}{\partial x_{p}}+A_{i p}^{(\gamma)} \frac{\partial v_{p}}{\partial x_{i}}+A_{p j}^{(\gamma)} \frac{\partial v_{p}}{\partial x_{i}} \\
(i, j=1,2,3 ; \gamma=1,2, \cdots, R-1) .
\end{gathered}
$$

In equations $(1.2), v_{i}(i=1,2,3)$ denote the velocity components in the $x_{i}$ coordinate system.

The coefficients in the tensor polynomial $P_{i i}$ are scalar invariants of the kinematic tensors, the coefficient of $A_{i j}^{(1)}$ vanishing when all the invariants vanish. The polynomial $P_{i j}$ may be assumed to have no term of degree zero, for any such term could be incorporated into the undetermined pressure $p$.

One type of flow considered in the previous paper was that generated solely by rigid walls in steady motion parallel to themselves, the fluid mass having no bounding surfaces other than rigid walls and no body forces being applied to the fluid. It was shown that flows of this type can be investigated quantitatively by the following procedure:

(i) A solution $\left(v_{\boldsymbol{i}}^{\prime}, p^{\prime}\right)$ to the equations of motion for an incompressible Newtonian fluid with viscosity $\mu$ is calculated neglecting inertia subject to the boundary condition that the fluid velocity at the walls match the velocity of the walls;

(ii) the solution $\left(v_{i}^{\prime}, p^{\prime}\right)$ calculated in $(i)$ is introduced into the equations of motion for the incompressible viscoelastic fluid described above, and the body forces required to support this solution are calculated neglecting inertia;

(iii) a solution $\left(v_{i}^{\prime \prime}, p^{\prime \prime}\right)$ to the equations of motion for an incompressible Newtonian fluid under the action of the body forces calculated in (ii) is calculated neglecting inertia, subject to the boundary condition of vanishing velocity on all the boundaries of the fluid mass;

*Received July 20, 1962; revised manuscript received Aug. 24, 1962.

**Throughout the paper, Latin indices except $r$ are tensor indices, Greek indices except $\theta$ and $\phi$ are running indices; $r, \theta$, and $\phi$ are reserved to denote the polar directions. 
(iv) then $\left(v_{i}=v_{i}^{\prime}-v_{i}^{\prime \prime}, p=p^{\prime}-p^{\prime \prime}\right)$ is an approximate solution to the equations of very slow motion for an incompressible, slightly viscoelastic fluid subject to the boundary condition of zero relative velocity at the walls. The error involved is of the second degree in $\epsilon$.

An example of this type of flow is the motion produced in a viscoelastic fluid contained between two concentric spheres which rotate slowly about a common axis of symmetry with different constant angular velocities.

If a Newtonian fluid is contained between the spheres and inertial effects are negligible, the streamlines of the flow are circles about the axis of rotation. Some aspects of the Newtonian flow with nonnegligible inertia have been considered by Proudman [2] and by Haberman [5].

It will be shown in the present paper that inclusion of viscoelastic terms in the equations of motion influences the flow between spheres in two distinct ways: some of these terms modify the distribution of the rotary flow about the axis, whereas others of them contribute to a secondary flow in the meridional planes.

2. The coordinate system. Let a spherical polar system $(r$. i; $p)$ be defined such that $r$ is the distance from the common center of the spheres, $r$ is ine colatitude measured from the axis of rotation, and $\phi$ is any longitude (see figure 1). It will sometimes be convenient to use the tensor notation

$$
r=x^{1}, \quad \theta=x^{2}, \quad \phi=x^{3} .
$$

The stress-deformation relation (1.1) becomes, in general tensor $n$ tation,

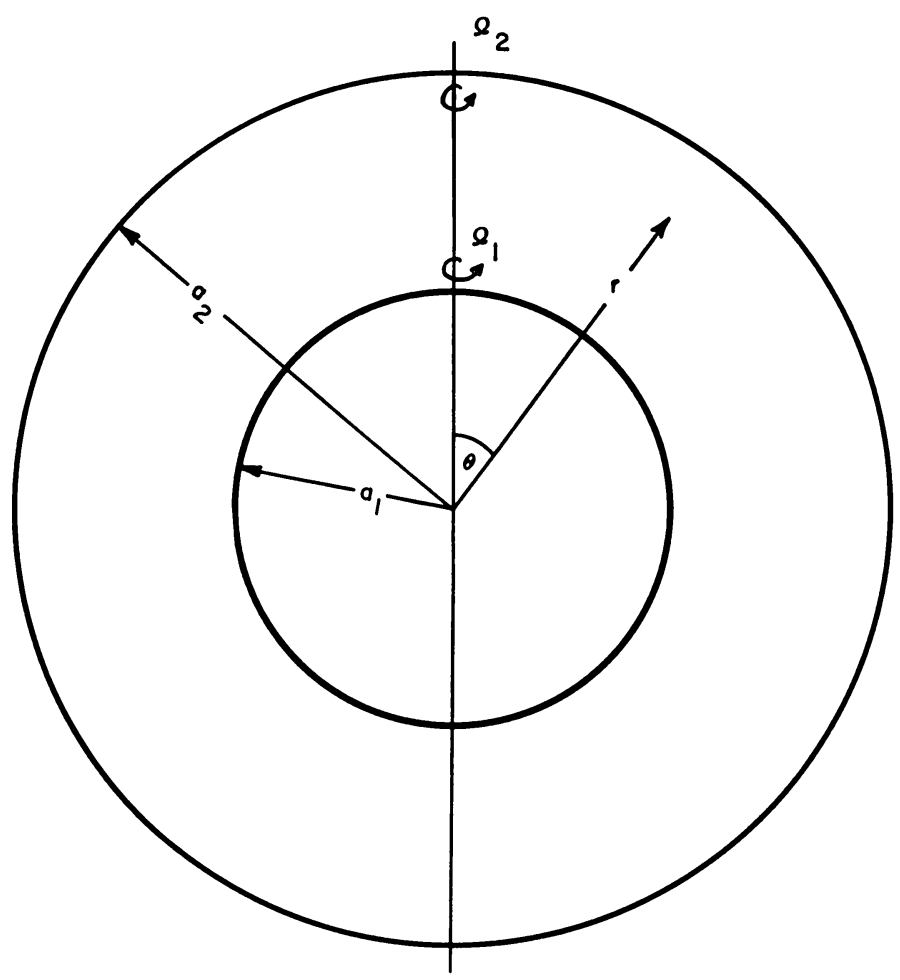

FIG. 1. The coordinate system. 


$$
t_{i}^{i}=-p \delta_{i}^{i}+\mu A_{i}^{(1) i}+\epsilon P_{i}^{i}(i, j=1,2,3),
$$

where $P_{j}^{i}$ is the same tensor polynomial function of the mixed kinematic tensors $A_{i}^{(\gamma) i}(\gamma=1,2, \cdots, R)$ that $P_{i j}$ is of the $A_{i j}^{(\gamma)}(\gamma=1,2, \cdots, R)$ in a Cartesian system.

The convariant kinematic tensors corresponding to the mixed tensors $A_{i}^{(\gamma) i}$ are determined by the expressions

$$
\begin{gathered}
A_{i j}^{(1)}=v_{i, j}+v_{i, i}(i, j=1,2,3), \\
A_{i j}^{(\gamma+1)}=v^{p} \frac{\partial A_{i j}^{(\gamma)}}{\partial x^{p}}+A_{i p}^{(\gamma)} \frac{\partial v^{p}}{\partial x^{j}}+A_{p i}^{(\gamma)} \frac{\partial v^{p}}{\partial x^{i}} \quad(i=1,2,3 ; \gamma=1,2, \cdots, R-1),
\end{gathered}
$$

where $v_{i}$ and $v^{i}(i=1,2,3)$ represent respectively the covariant and contravariant components of velocity in the $x^{i}$ system and commas denote covariant differentiation.

If the physical components of velocity in the spherical coordinate system $(r, \theta, \phi)$ are denoted by $V_{r}, V_{\theta}, V_{\phi}$, then

$$
\begin{aligned}
& v_{1}=v^{1}=V_{r}, \\
& v_{2}=r^{2} v^{2}=r V_{\theta}, \\
& v_{3}=r^{2} v^{3} \sin ^{2} \theta=r V_{\theta} \sin \theta .
\end{aligned}
$$

With the radius and angular velocity of the inner sphere denoted by $a_{1}$ and $\Omega_{1}$ respectively, and those of the outer sphere by $a_{2}$ and $\Omega_{2}$ respectively, the velocity components must satisfy the boundary conditions

$$
\begin{aligned}
& V_{r}\left(a_{1}, \theta, \phi\right)=V_{r}\left(a_{2}, \theta, \phi\right)=0, \\
& V_{\theta}\left(a_{1}, \theta, \phi\right)=V_{\theta}\left(a_{2}, \theta, \phi\right)=0, \\
& V_{\phi}\left(a_{1}, \theta, \phi\right)=a_{1} \Omega_{1} \sin \theta, \\
& V_{\phi}\left(a_{2}, \theta, \phi\right)=a_{2} \Omega_{2} \sin \theta .
\end{aligned}
$$

3. The Newtonian solution. If an incompressible Newtonian fluid of viscosity $\mu$ is contained betwen the spheres and if the motion is slow enough so that inertia can be neglected, the equations of motion

$$
\begin{gathered}
\mu\left(\nabla^{2} V_{r}-\frac{2}{r^{2}} V_{r}-\frac{2}{r^{2}} \frac{\partial V \theta}{\partial \theta}-\frac{2 V \theta \cot \theta}{r^{2}}\right)=\frac{\partial p}{\partial r}, \\
\mu\left(\nabla^{2} V \theta+\frac{2}{r^{2}} \frac{\partial V_{r}}{\partial \theta}-\frac{V \theta}{r^{2} \sin ^{2} \theta}\right)=\frac{1}{r} \frac{\partial p}{\partial \theta}, \\
\nabla^{2} V_{\phi}-\frac{V_{\phi}}{r^{2} \sin ^{2} \theta}=0,
\end{gathered}
$$

and the incompressibility condition

$$
r \frac{\partial V_{r}}{\partial r}+2 V_{r}+\frac{\partial V_{\theta}}{\partial \theta}+\cot \theta V_{\theta}=0
$$

must be satisfied at each point of the fluid mass. In equations (3.1),

$$
\nabla^{2}=\frac{\partial^{2}}{\partial r^{2}}+\frac{2}{r} \frac{\partial}{\partial r}+\frac{1}{r^{2}} \frac{\partial^{2}}{\partial \theta^{2}}+\frac{\cot \theta}{r^{2}} \frac{\partial}{\partial \theta} .
$$


A solution $\left(V_{r}^{\prime}, V_{\theta}^{\prime}, V_{\phi}^{\prime}, p^{\prime}\right)$ to equations (3.1) and (3.2), subject to the boundary conditions (2.6), is provided by

$$
V_{r}^{\prime}=0, \quad V_{\theta}^{\prime}=0, \quad V_{\phi}^{\prime}=\sin \theta\left(A r+B / r^{2}\right),
$$

with

$$
p^{\prime}=\text { constant. }
$$

The constants $A$ and $B$ in equations (3.4) are defined by

$$
A=\frac{a_{2}^{3} \Omega_{2}-a_{1}^{3} \Omega_{1}}{a_{2}^{3}-a_{1}^{3}}, \quad B=\frac{a_{1}^{3} a_{2}^{3}\left(\Omega_{1}-\Omega_{2}\right)}{a_{2}^{3}-a_{1}^{3}} .
$$

If the inner sphere is absent, the coefficient $B$ vanishes, so that equations (3.4) represent the rigid body motion obtained when a revolving sphere is completely filled with fluid. In this case, equations (3.4) described the motion even if the inertia terms are not neglected. (If inertia is not negligible the pressure is not constant, but is given by $p^{\prime}=$ constant $+\frac{1}{2} \rho A^{2} r^{2} \sin ^{2} \theta$.)

If the outer sphere is absent, the coefficient $A$ vanishes. Equations (3.4) then represent the flow generated in an infinite volume of fluid by a steadily revolving sphere. If the inertia terms are retained, equations (3.4) do not apply.

4. The body forces required to support the Newtonian solution in a viscoelastic fluid. The contravariant metric tensor $g^{i j}$ for the spherical coordinate system $(r, \theta, \phi)$ is given by

$$
\left\|g^{i i}\right\|=\left\|\begin{array}{ccc}
1, & 0, & 0 \\
0, & 1 / r^{2}, & 0 \\
0, & 0, & 1 /\left(r^{2} \sin ^{2} \theta\right)
\end{array}\right\| \text {. }
$$

From equations (2.3), (2.4), (2.5), it is then seen that, for the flow described by equations (3.4), the kinematic matrices $\mathbf{A}_{\gamma}=\left\|A_{i}^{(\gamma) i}\right\|(\gamma=1,2, \cdots, R)$ are given by

$$
\mathbf{A}_{1}=-\left(3 B / r^{4}\right) \mathbf{X}, \quad \mathbf{A}_{2}=\left(18 B^{2} \sin ^{2} \theta / r^{6}\right) \mathbf{Y}, \quad \mathbf{A}_{\boldsymbol{\gamma}}=0(\gamma \geq 3),
$$

where

$$
\begin{gathered}
\mathbf{X}=\left\|\begin{array}{ccc}
0, & 0, & r^{2} \sin ^{2} \theta \\
0, & 0, & 0 \\
1, & 0, & 0
\end{array}\right\| . \\
\mathbf{Y}=\left\|\begin{array}{lll}
1, & 0, & 0 \\
0, & 0, & 0 \\
0, & 0, & 0
\end{array}\right\| .
\end{gathered}
$$

It then follows from a result obtained by Rivlin [3] that the matrix $\mathbf{P}=\left\|P_{i}^{i}\right\|$ is expressible in the form

$$
\begin{aligned}
\mathbf{P}=\alpha_{1} \mathbf{A}_{1} & +\alpha_{2} \mathbf{A}_{2}+\alpha_{3} \mathbf{A}_{1}^{2}+\alpha_{4} \mathbf{A}_{2}^{2}+\alpha_{5}\left(\mathbf{A}_{1} \mathbf{A}_{2}+\mathbf{A}_{2} \mathbf{A}_{1}\right) \\
& +\alpha_{6}\left(\mathbf{A}_{1}^{2} \mathbf{A}_{2}+\mathbf{A}_{2} \mathbf{A}_{1}^{2}\right)+\alpha_{7}\left(\mathbf{A}_{1} \mathbf{A}_{2}^{2}+\mathbf{A}_{2}^{2} \mathbf{A}_{1}\right)+\alpha_{8}\left(\mathbf{A}_{1}^{2} \mathbf{A}_{2}^{2}+\mathbf{A}_{2}^{2} \mathbf{A}_{1}^{2}\right),
\end{aligned}
$$

where $\alpha_{\delta}(\delta=1,2, \cdots, 8)$ are polynomials in the invariants $\operatorname{tr} \mathbf{A}_{1}, \operatorname{tr} \mathbf{A}_{2}, \operatorname{tr} \mathbf{A}_{1}^{2}, \operatorname{tr} \mathbf{A}_{2}^{2}$, $\operatorname{tr} \mathbf{A}_{1}^{3}, \operatorname{tr} \mathbf{A}_{2}^{3}, \operatorname{tr} \mathbf{A}_{1}^{2} \mathbf{A}_{2}, \operatorname{tr} \mathbf{A}_{1} \mathbf{A}_{2}^{2}$, and $\operatorname{tr} \mathbf{A}_{1}^{2} \mathbf{A}_{2}^{2}, \alpha_{1}$ vanishing when all the invariants vanish. 
From equations (4.2),

$$
\begin{aligned}
\mathbf{A}_{1}^{2}=\frac{9 B^{2} \sin ^{2} \theta}{r^{6}}\left\|\begin{array}{lll}
1, & 0, & 0 \\
0, & 0, & 0 \\
0, & 0, & 1
\end{array}\right\|, \\
\mathbf{A}_{1} \mathbf{A}_{2}+\mathbf{A}_{2} \mathbf{A}_{1}=-\left(54 B^{3} \sin ^{2} \theta / r^{10}\right) \mathbf{X}, \\
\mathbf{A}_{2}^{2}=\mathbf{A}_{1}^{2} \mathbf{A}_{2}+\mathbf{A}_{2} \mathbf{A}_{1}^{2}=\left(324 B^{4} \sin ^{4} \theta / r^{12}\right) \mathbf{Y}, \\
\mathbf{A}_{1} \mathbf{A}_{2}^{2}+\mathbf{A}_{2}^{2} \mathbf{A}_{1}=-\left(972 B^{5} \sin ^{4} \theta / r^{16}\right) \mathbf{X}, \\
\mathbf{A}_{1}^{2} \mathbf{A}_{2}^{2}+\mathbf{A}_{2}^{2} \mathbf{A}_{1}^{2}=\left(5832 B^{6} \sin ^{6} \theta / r^{18}\right) \mathbf{Y} .
\end{aligned}
$$

The invariants become

$$
\begin{aligned}
& \operatorname{tr} \mathbf{A}_{1}=\operatorname{tr} \mathbf{A}_{1}^{3}=\operatorname{tr} \mathbf{A}_{1} \mathbf{A}_{2}=\operatorname{tr} \mathbf{A}_{1} \mathbf{A}_{2}^{2}=0, \\
& \operatorname{tr} \mathbf{A}_{2}=\operatorname{tr} \mathbf{A}_{1}^{2}=18 B^{2} \sin ^{2} \theta / r^{6}, \\
& \operatorname{tr} \mathbf{A}_{2}^{2}=2 \operatorname{tr} \mathbf{A}_{1}^{2} \mathbf{A}_{2}=324 B^{4} \sin ^{4} \theta / r^{12}, \\
& \operatorname{tr} \mathbf{A}_{2}^{3}=2 \operatorname{tr} \mathbf{A}_{1}^{2} \mathbf{A}_{2}^{2}=5832 B^{6} \sin ^{6} \theta / r^{18} .
\end{aligned}
$$

In view of equations (4.7), the coefficients $\alpha_{\delta}(\delta=1,2, \cdots, 8)$ are polynomials in $\left(B^{2} \sin ^{2} \theta / r^{6}\right)$, the coefficient $\alpha_{1}$ having no term in $\left(B^{2} \sin ^{2} \theta / r^{6}\right)^{0}$.

It then follows that equations for the physical stress components $\tau_{i j}(i, j=1,2,3)$, defined by

$$
\tau_{i i}=\left(\frac{g^{i j}}{g^{i i}}\right)^{1 / 2} t_{i}^{i} \quad \text { (not summed) }
$$

are given by

$$
\begin{aligned}
& \tau_{11}=-p^{\prime}+\epsilon \sum_{\delta=1}^{N}\left(A_{\delta}+B_{\delta}\right)\left(B \sin \theta / r^{3}\right)^{2 \delta}, \\
& \tau_{22}=-p^{\prime}, \\
& \tau_{33}=-p^{\prime}+\epsilon \sum_{\delta=1}^{N} B_{\delta}\left(B \sin \theta / r^{3}\right)^{2 \delta}, \\
& \tau_{23}=0, \\
& \tau_{31}=-3 B \mu \sin \theta / r^{3}+\epsilon \sum_{\delta=1}^{N} C_{\delta}\left(B \sin \theta / r^{3}\right)^{2 \delta+1}, \\
& \tau_{12}=0 .
\end{aligned}
$$

where $A_{\delta}, B_{\delta}, C_{\delta}(\delta=1,2, \cdots, N)$ are constants defined by the expansions

$$
\begin{aligned}
& 18 \alpha_{2} B^{2} \sin ^{2} \theta / r^{6}+324\left(\alpha_{4}+\alpha_{6}\right) B^{4} \sin ^{4} \theta / r^{12} \\
&+5832 \alpha_{8} B^{6} \sin ^{6} \theta / r^{18}=\sum_{\delta=1}^{N} A_{\delta}\left(B \sin \theta / r^{3}\right)^{2 \delta} \\
& 9 \alpha_{3} B^{2} \sin ^{2} \theta / r^{6}= \sum_{\delta=1}^{N} B_{\delta}\left(B \sin \theta / r^{3}\right)^{28}, \\
&-3 \alpha_{1}-54 \alpha_{5} B^{2} \sin ^{2} \theta / r^{6}-972 \alpha_{7} B^{4} \sin ^{4} \theta / r^{12}=\sum_{\delta=1}^{N} C_{\delta}\left(B \sin \theta / r^{3}\right)^{2 \delta} .
\end{aligned}
$$


The number $N$ of terms in each of these expansions and the magnitude of the coefficients $A_{\delta}, B_{\delta}, C_{\delta}(\delta=1,2, \cdots, N)$ depend on the form of the tensor polynomial $P_{i}^{i}$ for the material under consideration.

The body forces $\left(f_{\theta}^{\prime}, f_{r}^{\prime}, f_{\phi}^{\prime}\right)$ per unit mass of fluid required to support the flow described by equations (3.4) with $p=p^{\prime}$ are determined by the equilibrium equations

$$
\begin{aligned}
& -\rho f_{r}^{\prime}=\frac{\partial \tau_{11}}{\partial r}+\frac{1}{r} \frac{\partial \tau_{12}}{\partial \theta}+\frac{1}{r}\left(2 \tau_{11}-\tau_{22}-\tau_{33}+\cot \theta \tau_{12}\right), \\
& -\rho f_{\theta}^{\prime}=\frac{\partial \tau_{12}}{\partial r}+\frac{1}{r} \frac{\partial \tau_{22}}{\partial \theta}+\frac{1}{r}\left[\left(\tau_{22}-\tau_{33}\right) \cot \theta+3 \tau_{12}\right], \\
& -\rho f_{\phi}^{\prime}=\frac{\partial \tau_{31}}{\partial r}+\frac{1}{r} \frac{\partial \tau_{23}}{\partial \theta}+\frac{1}{r}\left(3 \tau_{31}+2 \tau_{23} \cot \theta\right) .
\end{aligned}
$$

Since $p^{\prime}$ is constant, substitution of equations (4.10) into equations (4.11) yields

$$
\begin{aligned}
& -\rho f_{r}^{\prime}=\frac{\epsilon}{r} \sum_{\delta=1}^{N}\left[B_{\delta}-2(3 \delta-1) A_{\delta}\right]\left(B \sin \theta / r^{3}\right)^{2 \delta}, \\
& -\rho f_{\theta}^{\prime}=\frac{\epsilon \cot \theta}{r} \sum_{\delta=1}^{N} B_{\delta}\left(B \sin \theta / r^{3}\right)^{2 \delta}, \\
& -p f_{\phi}^{\prime}=\frac{\epsilon}{r} \sum_{\delta=1}^{N} 3(2 \delta+1) C_{\delta}\left(B \sin \theta / r^{3}\right)^{2 \delta+1} .
\end{aligned}
$$

5. Calculation of the disturbance flow. The method described in the introduction can now be used to calculate the perturbation of the flow field caused by the viscoelasticity of the fluid. If a solution $\left(V_{r}^{\prime \prime}, V_{\theta}^{\prime \prime}, V_{\phi}^{\prime \prime}, p^{\prime \prime}\right)$ to the equations of motion for an incompressible Newtonian fluid under the action of the body force field described by equations (4.12) is calculated subject to the boundary conditions

$$
\begin{aligned}
& V_{r}^{\prime \prime}\left(a_{1}, \theta, \phi\right)=V_{r}^{\prime \prime}\left(a_{2}, \theta, \phi\right)=0, \\
& V_{\theta}^{\prime \prime}\left(a_{1}, \theta, \phi\right)=V_{\theta}^{\prime \prime}\left(a_{2}, \theta, \phi\right)=0, \\
& V_{\phi}^{\prime \prime}\left(a_{1}, \theta, \phi\right)=V_{\phi}^{\prime \prime}\left(a_{2}, \theta, \phi\right)=0,
\end{aligned}
$$

then $\left(V_{r}=V_{r}^{\prime}-V_{r}^{\prime \prime}, V_{\theta}=V_{\theta}^{\prime}-V_{\theta}^{\prime \prime}, V_{\phi}=V_{\phi}^{\prime}-V_{\phi}^{\prime \prime}, p=p^{\prime}-p^{\prime \prime}\right)$ is an approximate solution (with an error of the second degree in $\epsilon$ ) for the flow between the spheres of a fluid having the stress-deformation relation (1.1).

The disturbance flow $\left(V_{r}^{\prime \prime}, V_{\theta}^{\prime \prime}, V_{\phi}^{\prime \prime}, p^{\prime \prime}\right)$ should, therefore, be chosen to satisfy the equations of motion

$$
\begin{gathered}
\mu\left(\nabla^{2} V_{r}^{\prime \prime}-\frac{2}{r^{2}} V_{r}^{\prime \prime}-\frac{2}{r^{2}} \frac{\partial V_{\theta}^{\prime \prime}}{\partial \theta}-\frac{2 V_{\theta}^{\prime \prime} \cot \theta}{r^{2}}\right)=\frac{\partial p_{-}^{\prime \prime}}{\partial r}-\rho f_{r}^{\prime}, \\
\mu\left(\nabla^{2} V_{\theta}^{\prime \prime}+\frac{2}{r^{2}} \frac{\partial V_{r}^{\prime \prime}}{\partial \theta}-\frac{V \theta^{\prime \prime}}{r^{2} \sin ^{2} \theta}\right)=\frac{1}{r} \frac{\partial p^{\prime \prime}}{\partial \theta}-\rho f_{\theta}, \\
\mu\left(\nabla^{2} V_{\phi}^{\prime \prime}-\frac{V_{\phi}^{\prime \prime}}{r^{2} \sin ^{2} \theta}\right)=-\rho f_{\phi}^{\prime},
\end{gathered}
$$


and the incompressibility condition

$$
r \frac{\partial V_{r}^{\prime \prime}}{\partial r}+2 V_{r}^{\prime \prime}+\frac{\partial V_{\theta}^{\prime \prime}}{\partial \theta}+\cot \theta V_{\theta}^{\prime \prime}=0
$$

subject to the boundary conditions (5.1) to (5.3).

If a stream function $\Psi(r, \theta)$ is introduced such that

$$
\begin{aligned}
& V_{r}^{\prime \prime}=\frac{1}{r^{2} \sin \theta} \frac{\partial \Psi}{\partial \theta}, \\
& V_{\theta}^{\prime \prime}=-\frac{1}{r \sin \theta} \frac{\partial \Psi}{\partial r},
\end{aligned}
$$

the incompressibility condition (5.7) is automatically satisfied. Also, $p^{\prime \prime}$ can be eliminated from equations (5.4) and (5.5) by cross-differentiation, giving the differential equation for the stream function

$$
E^{4} \Psi=\frac{\rho}{\mu}\left[\frac{\partial}{\partial r}\left(r f_{\theta}^{\prime}\right)-\frac{\partial f_{r}^{\prime}}{\partial \theta}\right] \sin \theta
$$

where

$$
E^{4}=\left(\frac{\partial^{2}}{\partial r^{2}}-\frac{\cot \theta}{r^{2}} \frac{\partial}{\partial \theta}+\frac{1}{r^{2}} \frac{\partial^{2}}{\partial \theta^{2}}\right)^{2}
$$

Since by equations (5.1) and (5.2) both $V_{r}^{\prime \prime}$ and $V_{\theta}^{\prime \prime}$ vanish at $r=a_{1}$ and at $r=a_{2}$, since there can be no flow across the axis of symmetry, and since an additive constant does not affect the physical significance of a stream function, the boundary conditions to be imposed upon $\Psi$ may be taken as

$$
\Psi\left(a_{1}, \theta\right)=\Psi\left(a_{2}, \theta\right)=\frac{\partial \Psi\left(a_{1}, \theta\right)}{\partial r}=\frac{\partial \Psi\left(a_{2}, \theta\right)}{\partial r}=0 .
$$

By a substitution of the expressions (4.12) for the components of body force into equations (5.6) and (5.9), the problem of calculating the disturbance flow is reduced to the problem of integrating the partial differential equations

$$
\begin{gathered}
\left(\nabla^{2}-\frac{1}{r^{2} \sin ^{2} \theta}\right) V_{\phi}^{\prime \prime}=\frac{\epsilon}{\mu r} \sum_{\delta=1}^{N} 3(2 \delta+1) C_{\delta}\left(B \sin \theta / r^{3}\right)^{2 \delta+1}, \\
E^{4} \Psi=-\frac{\epsilon \cos \theta}{\mu r} \sum_{\delta=1}^{N} 4 \delta\left[(3 \delta-1) A_{\delta}+B_{\delta}\right]\left(B \sin \theta / r^{3}\right)^{2 \delta}
\end{gathered}
$$

subject to the boundary conditions (5.3) and (5.11), respectively.

The velocity component $V_{\phi}^{\prime \prime}$ can be determined with the aid of the identity

$$
\begin{array}{r}
{\left[\nabla^{2}-\frac{1}{r^{2} \sin ^{2} \theta}\right]\left[f(r) \sin ^{2 \delta+1} \theta\right]=\sin ^{2 \delta+1} \theta\left[\frac{d^{2}}{d r^{2}}+\frac{2}{r} \frac{d}{d r}-\frac{2(\delta+1)(2 \delta+1)}{r^{2}}\right] f(r)} \\
+4 \delta(\delta+1) \frac{\sin ^{2 \delta-1} \theta}{r^{2}} f(r),
\end{array}
$$

which is valid for any twice differentiable function $f(r)$. 
With this identity, it is apparent that

$$
V_{\phi}^{\prime \prime}=\frac{\epsilon}{\mu} \sum_{\delta=0}^{N} f_{\delta}(r) \sin ^{2 \delta+1} \theta
$$

is a solution to equation (5.12) subject to the boundary conditions (5.3), provided the functions $f_{\delta}(r)(\delta=0,1, \cdots, N)$ are chosen to satisfy the system of ordinary differential equations

$$
\begin{aligned}
& {\left[\frac{d^{2}}{d r^{2}}+\frac{2}{r} \frac{d}{d r}-\frac{2(N+1)(2 N+1)}{r^{2}}\right] f_{N}(r)=\frac{3(2 N+1) C_{N} B^{2 N+1}}{r^{6 N+4}},} \\
& {\left[\frac{d^{2}}{d r^{2}}+\frac{2}{r} \frac{d}{d r}-\frac{2(\delta+1)(2 \delta+1)}{r^{2}}\right] f_{\delta}(r)+\frac{4(\delta+1)(\delta+2)}{r^{2}} f_{\delta+1}(r)} \\
& =\frac{3(2 \delta+1) C_{\delta} B^{2 \delta+1}}{r^{6 \delta+4}}(\delta=1,2, \cdots, N-1 ; N \geq 2), \\
& {\left[\frac{d^{2}}{d r^{2}}+\frac{2}{r} \frac{d}{d r}-\frac{2}{r^{2}}\right] f_{0}(r)+\frac{8}{r^{2}} f_{1}(r)=0,}
\end{aligned}
$$

subject to the boundary conditions

$$
f_{\delta}\left(a_{1}\right)=f_{\delta}\left(a_{2}\right)=0(\delta=0,1, \cdots, N) .
$$

For $N=1$, only the first and last of equations (5.16) are used.

For a specified value of $N$, equations (5.16) can be integrated in succession, beginning with the equation involving only $f_{N}(r)$. Each stage of the process involves the integration of a second order ordinary differential equation of the Euler type. Since only one of the unknown functions $f_{\delta}(r)(\delta=0,1, \cdots, N)$ is determined at each step, this is not a difficult task.

In a similar manner, the identity

$$
\begin{aligned}
& E^{4}\left[f(r) \sin ^{2 \delta} \theta \cos \theta\right]=\sin ^{2 \delta} \theta \cos \theta\left[\frac{d^{2}}{d r^{2}}-\frac{2 \delta(2 \delta+1)}{r^{2}}\right]^{2} f(r) \\
& \quad+8 \delta(\delta-1) \sin ^{2(\delta-1)} \theta \cos \theta\left[\frac{1}{r^{2}} \frac{d^{2}}{d r^{2}}-\frac{2}{r^{3}} \frac{d}{d r}-\frac{2(\delta-1)(2 \delta+1)}{r^{4}}\right] f(r) \\
& \quad+16 \delta(\delta-1)^{2}(\delta-2) \frac{\sin ^{2(\delta-2)} \theta \cos \theta}{r^{4}} f(r),
\end{aligned}
$$

which is valid for any function $f(r)$ with four derivatives, facilitates the determination of the stream function $\Psi$.

A solution to equation (5.13) subject to the boundary conditions (5.11) is

$$
\Psi=-\frac{\epsilon \cos \theta}{\mu r} \sum_{\delta=1}^{N} g \delta(r) \sin ^{2 \delta} \theta,
$$

where the functions $g_{\delta}(r)(\delta=1,2, \cdots, N)$ are chosen to satisfy the ordinary differential equations 


$$
\begin{aligned}
& {\left[\frac{d^{2}}{d r^{2}}-\frac{2 N(2 N+1)}{r^{2}}\right]^{2} g_{N}(r)=4 N\left[(3 N-1) A_{N}+B_{N}\right] B^{2 N} / r^{6 N},} \\
& {\left[\frac{d^{2}}{d r^{2}}-\frac{2(N-1)(2 N-1)}{r^{2}}\right]^{2} g_{N-1}(r)} \\
& \quad+8 N(N-1)\left[\frac{1}{r^{2}} \frac{d^{2}}{d r^{2}}-\frac{2}{r^{3}} \frac{d}{d r}-\frac{2(N-1)(2 N+1)}{r^{4}}\right] g_{N}(r) \\
& =4(N-1)\left[(3 N-4) A_{N-1}+B_{N-1}\right] B^{2(N-1)} / r^{6(N-1)}, \\
& {\left[\frac{d^{2}}{d r^{2}}-\frac{2 \delta(2 \delta+1)}{r^{2}}\right]^{2} g_{\delta}(r) \quad} \\
& +8 \delta(\delta+1)\left[\frac{1}{r^{2}} \frac{d^{2}}{d r^{2}}-\frac{2}{r^{3}} \frac{d}{d r}-\frac{2 \delta(2 \delta+3)}{r^{4}}\right] g_{\delta+1}(r) \\
& +\frac{16 \delta(\delta+1)^{2}(\delta+2)}{r^{4}} g_{\delta+2}(r)=4 \delta\left[(3 \delta-1) A_{\delta}+B_{\delta}\right] B^{2 \delta} / r^{6 \delta}
\end{aligned}
$$

subject to the boundary conditions

$$
g_{\delta}\left(a_{1}\right)=g_{\delta}\left(a_{2}\right)=\frac{d g_{\delta}\left(a_{1}\right)}{d r}=\frac{d g_{\delta}\left(a_{2}\right)}{d r}=0(\delta=1,2, \cdots, N) .
$$

For $N=1$, only the first of equations (5.20) are used; for $N=2$, the first two equations are used.

For a specified value of $N$, integration of equations (5.20) in succession, beginning with the equation involving only $f_{N}(r)$, yields at each stage an ordinary, fourth order Euler differential equation in one of the unknown functions $g_{\delta}(r)(\delta=1,2, \cdots, N)$.

6. Illustration of a special case. It is seen from equations (5.12) that if one or more of the $C_{\delta}(\delta=1,2, \cdots, N)$ be different from zero, the distribution of the aximuthal velocity component $V_{\phi}=V_{\phi}-V_{\phi}^{\prime \prime}$ is different from that which is found in a Newtonian fluid.

A perhaps more striking effect is observed if one or more of the coefficients $\left[(3 \delta-1) A_{\delta}+B_{\delta}\right](\delta=1,2, \cdots, N)$ appearing in equations (5.13) differ from zero. In this case, a secondary circulation in the meridional planes is found. This circulation, which is transverse to the main (rotary) flow, occurs in a Newtonian fluid only as an effect of inertia. In a viscoelastic material, however, it can occur under conditions wherein the inertial terms are definitely negligible. In particular, it is found if the stress-deformation relation for the fluid is given by

$$
t_{i}^{i}=-p \delta_{i}^{i}+\mu A_{i}^{(1) i}+\epsilon\left(\lambda A_{k}^{(1) i} A_{i}^{(1) k}+\nu A_{i}^{(2) i}\right),
$$

where $\lambda$ and $\nu$ are constants $(\lambda \neq-4 \nu)$.

For this case,

$$
N=1, A_{1}=18 \nu, B_{1}=9 \lambda, C_{1}=0 .
$$

Since $N=1$ and $C_{1}=0$, equation (5.12) and the boundary conditions (5.3) imply that $V_{\phi}^{\prime \prime}=0$, so that $V_{\phi}=V_{\phi}^{\prime}$ as given by the third of equations (3.4). 


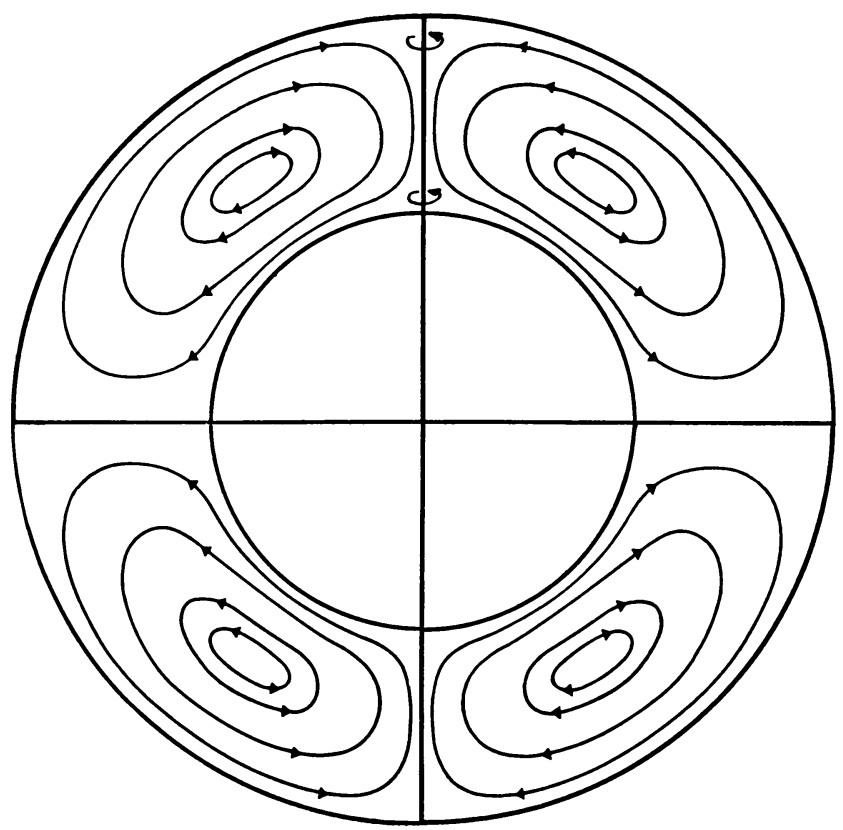

Fig. 2. The secondary flow.

Equation (5.19) for the stream function $\Psi$ becomes

$$
\Psi=-\frac{\epsilon}{\mu r} g_{1}(r) \cos \theta \sin ^{2} \theta,
$$

where $g_{1}(r)$ satisfies the ordinary differential equation

$$
\left[\frac{d^{2}}{d r^{2}}-\frac{6}{r^{2}}\right]^{2} g_{1}(r)=36(4 \nu+\lambda) B^{2} / r^{7},
$$

subject to the boundary conditions

$$
g_{1}\left(a_{1}\right)=g_{1}\left(a_{2}\right)=\frac{d g_{1}\left(a_{1}\right)}{d r}=\frac{d g_{1}\left(a_{2}\right)}{d r}=0 .
$$

The general solution to equation (6.4) is

$$
g_{1}(r)=\frac{B^{2}}{4}(4 \nu+\lambda)\left(\frac{1}{r^{3}}+\frac{a}{r^{2}}+b+c r^{3}+d r^{5}\right),
$$

where $a, b, c, d$ are constants. The boundary conditions (6.5) are satisfied by choosing these constants so that they satisfy the four linear equations

$$
\begin{gathered}
b+a / a_{1}^{2}+c a_{1}^{3}+d a_{1}^{5}=-1 / a_{1}^{3}, \\
b+a / a_{2}^{2}+c a_{2}^{3}+d a_{2}^{5}=-1 / a_{2}^{3}, \\
-2 a / a_{1}^{3}+3 c a_{1}^{2}+5 d a_{1}^{4}=3 / a_{1}^{4}, \\
-2 a / a_{2}^{3}+3 c a_{2}^{3}+5 d a_{2}^{4}=3 / a_{2}^{4} .
\end{gathered}
$$


The secondary flow having the stream function $\Psi$ given by equation (6.3), with $g_{1}(r)$ given by equation (6.6), is illustrated in Figure 2 for $a_{2}=2 a_{1}$ with $(4 \nu+\lambda)$ positive. The direction of flow is reversed for $(4 \nu+\lambda)$ negative.

\section{REFERENCES}

1. W. E. Langlois and R. S. Rivlin, Steady flow of slightly viscoelastic fluids, Brown University Technical Report No. 3, Contract DA-19-020-ORD-4725, 1959

2. I. Proudman, The almost-rigid rotation of viscous fluid between concentric spheres, J. Fluid Mechanics 1, 505 (1956)

3. R. S. Rivlin, Further remarks on the stress-deformation relations for isotropic materials, J. Ratl. Mech. Anal. 4, 681 (1955)

4. R. S. Rivlin and J. L. Ericksen, Stress-deformation relations for isotropic materials, J. Ratl. Mech. Anal. 4, 323 (1955)

5. W. L. Haberman, Secondary flow about a sphere rotating in a viscous liquid inside a coaxially rotating spherical container, Phys. of Fluids 5, 625 (1962) 